\title{
Detection and Analysis of Water Vapor Transport by Airborne Lidars
}

\author{
Christoph Kiemle, Andreas Schäfler, Martin Wirth, Andreas Fix, and Stephan Rahm
}

\begin{abstract}
Water vapor, a minor constituent of the earth's atmosphere, plays a major role in the radiation budget and the water cycle with important implications for weather and climate. Due to the heterogeneous distribution of its sources, evaporation, and sinks, condensation and precipitation, and due to the complexity of atmospheric motion and mixing, its distribution in the atmosphere is highly variable. Despite the high relevance of this trace gas, its variability challenges accurate measurements of its concentration. Advanced airborne lidar instruments aid to better observe water vapor and its transport in the atmosphere, in view of an improved understanding of atmospheric processes that are key to weather and climate research. The combination of a water vapor differential absorption lidar and a heterodyne detection Doppler wind lidar on an aircraft is new and allows to measure vertical profiles of the latent heat flux in a convective boundary layer and to portray the small- to large-scale humidity transport and variability along the aircraft flight track with high accuracy and spatial resolution.
\end{abstract}

Index Terms-Airborne lidar, boundary layer, differential absorption, latent heat flux, water vapor transport.

\section{INTRODUCTION}

W ATER vapor is routinely measured at surface stations, with the global radiosonde network and with satellite sensors. The radiosondes' accuracy is sufficient in the lower and middle troposphere, but the representativeness of the one-dimensional vertical profiles is poor. Furthermore, few data are collected over the oceans, at high latitudes, and in the southern hemisphere. In these regions, and in the upper troposphere, passive satellite spectrometers partly fill the observational gap, but with coarse spatial resolution. Better accuracy and resolution is obtained by active, optical lidar remote sensing. Since 1987 the lidar group of the DLR institute for atmospheric physics develops airborne water vapor lidars. Participation in numerous field experiments helped characterize the distribution of water vapor in the frame of case studies dedicated to specific physical and meteorological processes. A recent innovative highlight is the co-deployment of a newly developed water vapor and a wind lidar onboard the DLR Falcon research aircraft (Fig. 1; Table I).

The differential absorption lidar (DIAL) method uses the difference of atmospheric backscatter signals from a laser emitting on a molecular $\mathrm{H}_{2} \mathrm{O}$ absorption line, and a non-absorbed off-line reference laser. The signal difference is proportional to

Manuscript received September 28, 2012; revised December 21, 2012; accepted December 25, 2012. Date of publication January 25, 2013; date of current version June 17, 2013.

The authors are with the Lidar Group, Institut für Physik der Atmosphäre, Deutsches Zentrum für Luft- und Raumfahrt (DLR), 82234 Oberpfaffenhofen, Germany.

Color versions of one or more of the figures in this paper are available online at http://ieeexplore.ieee.org.

Digital Object Identifier 10.1109/JSTARS.2013.2239609

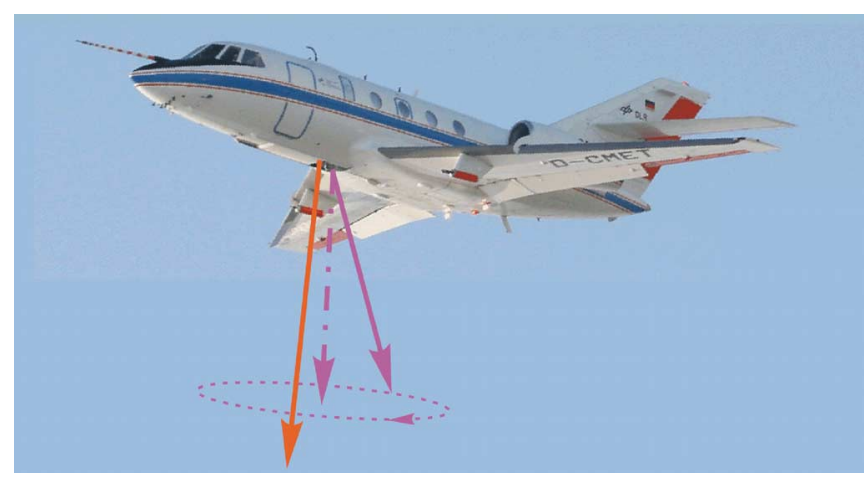

Fig. 1. DLR Falcon research aircraft with sketch of wind (pink) and water vapor (orange) lidar beams. The wind lidar can be operated either in conical scanning mode for measurements of the three-dimensional wind vector, or in nadir viewing mode for vertical wind velocity profiles.

TABLE I

MAIN CHARACTERISTICS OF AIRBORNE WATER VAPOR DifFERENTIAL ABSORPTION LIDAR (DIAL) AND DOPPLER WIND LIDAR

\begin{tabular}{lll} 
& DIAL & Wind Lidar \\
\hline$==================$ \\
Transmitter type & OPO & Tm:LuAG \\
Wavelength $(\mathrm{nm})$ & 935 & 2022 \\
Pulse energy $(\mathrm{mJ})$ & 40 & 1.5 \\
PRF $(\mathrm{Hz})$ & 200 & 500 \\
Average power $(\mathrm{W})$ & 8 & 0.75 \\
Detection principle & direct & heterodyne \\
Detector type & APD & PIN diode \\
Telescope diam. $(\mathrm{cm})$ & 48 & 10 \\
Precision & $0.4 \mathrm{~g} / \mathrm{kg}$ & $0.1 \mathrm{~m} / \mathrm{s}$ \\
$========================$
\end{tabular}

the $\mathrm{H}_{2} \mathrm{O}$ molecule density in air. The DLR DIAL has two laser transmitters, each based on an injection-seeded optical parametric oscillator (OPO) pumped by the second harmonic of a Q-switched, diode-pumped single-mode Nd:YAG laser at a pulse repetition frequency (PRF) of $100 \mathrm{~Hz}$ [1]. The OPO is optimized to operate in the spectral region between $920-950 \mathrm{~nm}$ at an average output pulse energy of $40 \mathrm{~mJ}$ (Table I). Each transmitter outputs two spectrally narrow pulses, yielding in total four pulses (each $50 \mathrm{~Hz}, 40 \mathrm{~mJ}$ ) at different wavelengths to probe four water vapor absorption lines of different strength. This allows obtaining vertical profiles throughout the troposphere and the lower stratosphere, layers in which the water vapor concentrations can vary over four orders of magnitude.

The wind lidar uses the Doppler Effect along the line-of-sight of the laser beam. It is operated either in conical-scanning mode 


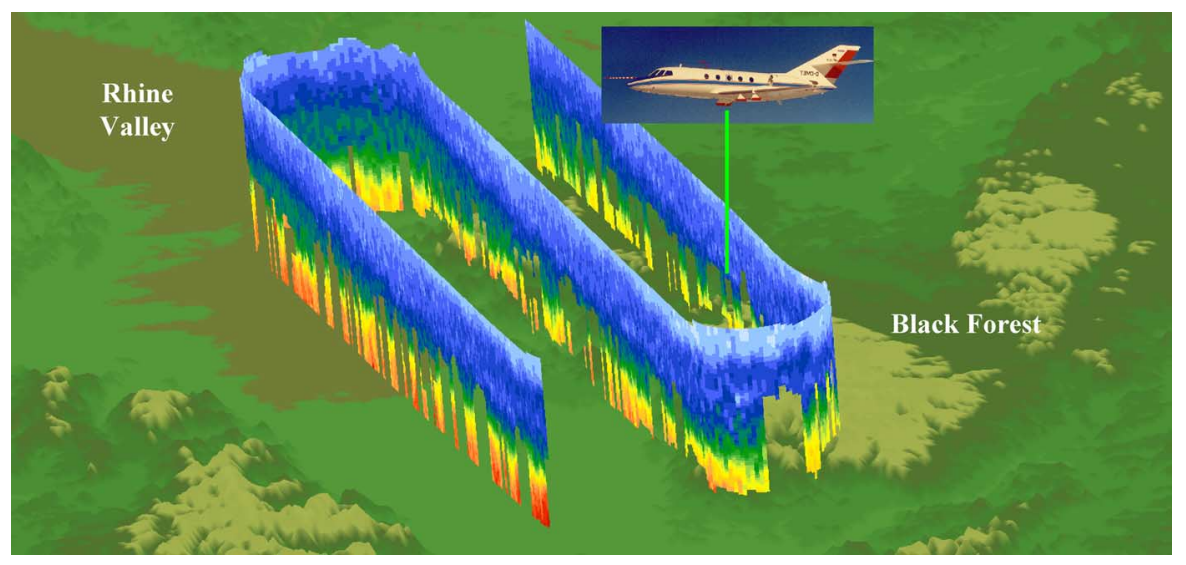

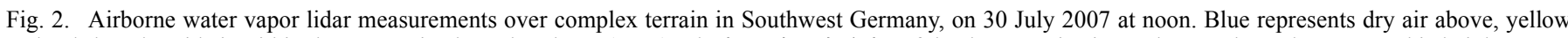

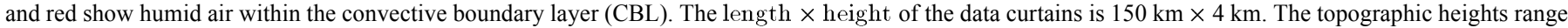
from $150 \mathrm{~m}$ to $1500 \mathrm{~m}$. Interruptions are due to scattered fair-weather clouds at the CBL top.

with $20^{\circ}$ off-nadir angle for 3 -d wind field profiles, or in nadirviewing mode for vertical wind speed profiles beneath the aircraft flight track. Examples for both measurement modes are given in the following two sections.

\section{Vertical Water VAPOR Transport, LATENT Heat FluX}

Evaporation over land and especially over complex, mountainous terrain can be very heterogeneous on small scales that are both under-sampled by observations, and un-resolved by numerical weather prediction (NWP) models. Over a land surface heated by solar irradiation, a convective boundary layer (CBL) develops, typically reaching heights of $1-2 \mathrm{~km}$ above ground in the afternoon. The buoyancy of warm air parcels transports the humidity vertically away from its surface sources. Cloud condensation at the boundary layer top, and subsequent cloud growth with occasionally heavy precipitation may occur. Observational and modeling deficits due to the heterogeneity of surface and atmospheric moisture are main reasons why numerical weather prediction (NWP) models often fail to accurately forecast the precipitation in the warm season, even though evaporation and subsequent humidity transport are key elements of the hydrological cycle.

Consequently, a field experiment called "Convective and Orographically-induced Precipitation Study" (COPS) was conducted in July 2007 over the Black Forest low-mountains in south-western Germany, with the participation of the DLR Falcon aircraft. Fig. 2 exemplarily shows two-dimensional vertical cross-sections of humidity beneath the aircraft along the flight track. This kind of data gives a decisive advantage over conventional one-dimensional "in-situ" measurements, particularly in this heterogeneous situation, where e.g., higher humidity is observed in the Rhine valley. One of the main goals of the DLR Falcon was to measure the vertical humidity transport within the CBL over the Rhine valley and the Black Forest on days when thunderstorms, mainly influenced by the orography and surface humidity, were forecasted [2].

Fig. 3 gives an overview of the lidar measurements of aerosol backscatter, vertical velocity, and humidity. The DIAL offline signals' backscatter intensity displays the CBL complexity and the clouds forming at the CBL top (black). The backscatter contrast with the free troposphere above provides a precise indication of the instantaneous, irregular CBL top. The Doppler wind lidar profiles accurately reproduce the convective up- and downdrafts of varying size and strength. There is no vertical wind in the free troposphere above the CBL. The DIAL humidity plot shows the contrast between the CBL with $>4 \mathrm{~g} / \mathrm{kg}$ over this flight leg and the above drier free troposphere at $\sim 2 \mathrm{~g} / \mathrm{kg}$ water vapor mass mixing ratio. White areas are gaps due to clouds shadowing the lidar signals.

From the lidar-sensed wind and water vapor fluctuations in the convective boundary layer, a representative area-averaged latent heat flux profile is obtained by the eddy-covariance technique [2]: humid updrafts, as well as dry downdrafts, produce a net upward-directed transport of moisture, i.e., a positive flux of latent heat, as was observed here in this case. What is latent heat? The liquid phase of water has strong intermolecular bonds.

Evaporation, the transition to the gas phase, requires a considerable amount of energy to free these bonds. This energy is called latent heat: Heat is released when water vapor condenses into clouds or onto surfaces, and cools the air when condensate (cloud or rain droplets) evaporates. A significant portion of the energy in extreme weather events such as fronts, thunderstorms and cyclones is provided by the latent heat released when the associated large clouds form and grow.

In Fig. 3, a few strong thermals, well visible in the vertical velocity profiles, appear responsible for most of the flux. Their locations are not generally found above "hot spots" as one would expect, because horizontal winds may shift and blur the patterns of surface hot spots. Similarly, the variability of vertical wind, moisture and the CBL top seems unrelated to surface features such as the city of Strasbourg overflown close to $48.6^{\circ}$ N. Fig. 3 finally shows that the separation of atmospheric variability, surface influences and turbulent transport is not straightforward. Nevertheless, the lidars' spatial, horizontal and vertical resolution is $200 \mathrm{~m}$, is found to be sufficient to resolve the dominant contributions to the flux of latent heat, since co-spectra of the humidity and vertical velocity measurements show a distinct peak at wavelengths between 1 and $4 \mathrm{~km}$, which indicates that the relevant contributions to the net flux occur on these spatial scales. 
The lidar-derived latent heat fluxes over the region of Fig. 3 and the mountains displayed in Fig. 2 vary between $100-500 \mathrm{~W} / \mathrm{m}^{2}$. The meteorological interpretation is that these positive fluxes moistened the growing CBL by upward transport of humidity from surface evaporation due to previous days' rain. They were however too low to generate the thunderstorms that had been predicted to occur with small probability on this particular day. To conclude, the unique, airborne double-lidar instrumentation proves to be a valuable new tool for the study of various important CBL processes.

\section{HORIZONTAL WATER VAPOR TRANSPORT}

In the mid-latitudes between 30 and $60^{\circ} \mathrm{N}$, cyclones effectively remove energy differences between polar and subtropical areas as a result of differing solar radiation by transporting moist and warm air pole-ward and cold and dry air equator-ward. Typically, the polar and subtropical air masses are separated by a narrow band in the mid-latitudes possessing a sharp meridional temperature change. Along this frontal zone, low pressure systems evolve that transport air horizontally and vertically, generating cloud development and precipitation. Due to the minor depth of the layer where weather happens, the troposphere $(0-12$ $\mathrm{km}$ ), most atmospheric transport processes can be considered quasi-horizontal. Advanced observation techniques like lidar are required to observe the water vapor transport in low pressure systems. Besides the understanding of the physical processes, the verification of model simulations by independent observations helps pinpointing insufficiencies in current NWP models.

Fig. 4 shows an example from the COPS field experiment to highlight the value of high resolution lidar data to observe the highly-complex three-dimensional transport of moisture in the surrounding of a low pressure system. The curtain-like two-dimensional cross section represents a snapshot of the water vapor transport, determined by the product of wind velocity and water vapor concentration, and reveals an up to $6 \mathrm{~km}$ deep layer of enhanced water vapor transport in the warm sector of the cyclone [3].

Maximum values occur on the most western part of the flight facing the cold front, where increased wind speeds and water vapor concentrations coincide. Nicely, the strong horizontal gradients of the moisture transport at the warm front show a tilting with height, coming along with the northeastward ascending air masses (red dashed lines along the lidar cross section). The tilting is observed on both overpasses of the warm front. Red arrows denote the up-gliding of the warm and moist air above the colder air to the north. In contrast, in the north-eastern part the horizontal transport in the boundary layer is weak.

Fig. 4(b) shows the same scenery from a northwestern perspective. The increased transport on the western side that extends upward along the tilted warm front is clearly visible. The figure also displays trajectories that describe the pathways of the observed air parcels in space and time. Shown are the pathways up to 2.5 days in advance and up to 1.5 days after the lidar observation. The trajectories were calculated using NWP wind data. The strong moisture transport clearly originates from the southwest. Air in the lower troposphere is humidified over the Atlantic and the Mediterranean by evaporation and subsequent vertical transport in the boundary layer. These air masses converge over southeastern Europe. The developing cyclone incorporates and transports the moisture northeastward towards the flight path. Many air parcels slightly ascend towards the lidar cross section and further up until they reach the upper troposphere $(\sim 8 \mathrm{~km}) 1.5$ days after being observed by the lidar. An in-depth discussion of this case study and a comparison with NWP data is found in [3].

The measurement results demonstrate that the airborne lidar instruments are able to map the clear-air transport of water vapor into cyclones that is known to play a key role in the structure, intensity, and further evolution of cyclones. The latent heat release is expected to amplify cyclones, and also to affect the upperlevel wind fields which control the weather development further downstream. This emphasizes the importance of a correct representation of moisture for improving the quality of weather forecasts. Further studies show that the lidar observations are useful for diagnosing NWP model errors which result from either incorrect horizontal or vertical transport of moisture or from insufficiently represented evaporation in moisture source regions.

\section{CONCLUSION AND Discussion}

Accurate water vapor measurements are sparse and challenging because of the high variability of its sources in space and time, and because of the complexity of atmospheric motion and mixing. Advanced airborne water vapor observations are, as shown, valuable for addressing relevant issues of current research in atmospheric water vapor and transport. Previous inter-comparison studies with other instruments have shown that the DLR DIAL water vapor profiles are highly accurate throughout the troposphere and also in the lower stratosphere [4], [5].

There is an increasing demand for improved measurements, for example in the upper troposphere in regions with ice supersaturation, where persistent cirrus clouds may form from contrails after the passage of an aircraft. Here, measurements of the relative humidity are important. This implies having simultaneous information on temperature from NWP data or auxiliary measurements. A combined water vapor and temperature lidar would be a future challenging task, as temperature profiling by lidar is basically possible yet still subject to high detection noise, which necessitates long averaging periods and consequently means coarse spatial resolution.

Fig. 5 shows the various and manifold interactions of water vapor in the earth's atmosphere. Water vapor in the lower troposphere is particularly relevant for weather, while in the upper troposphere and the stratosphere it is particularly relevant for climate, where, after vertical transport from the surface sources, it strongly influences Earth's radiation budget. Consequently, understanding the transport and distribution of atmospheric humidity and the processes that control it is of primary importance to improve both climate research and weather prediction.

New instrument developments such as multi-wavelength and combined lidar will, on board the new German HALO (high-altitude long-range) research aircraft, advance the science, building on the knowledge gained in the above-mentioned experiments. Foreseen is e.g., the simultaneous integration of ozone and water vapor lidars to study exchange processes of air 


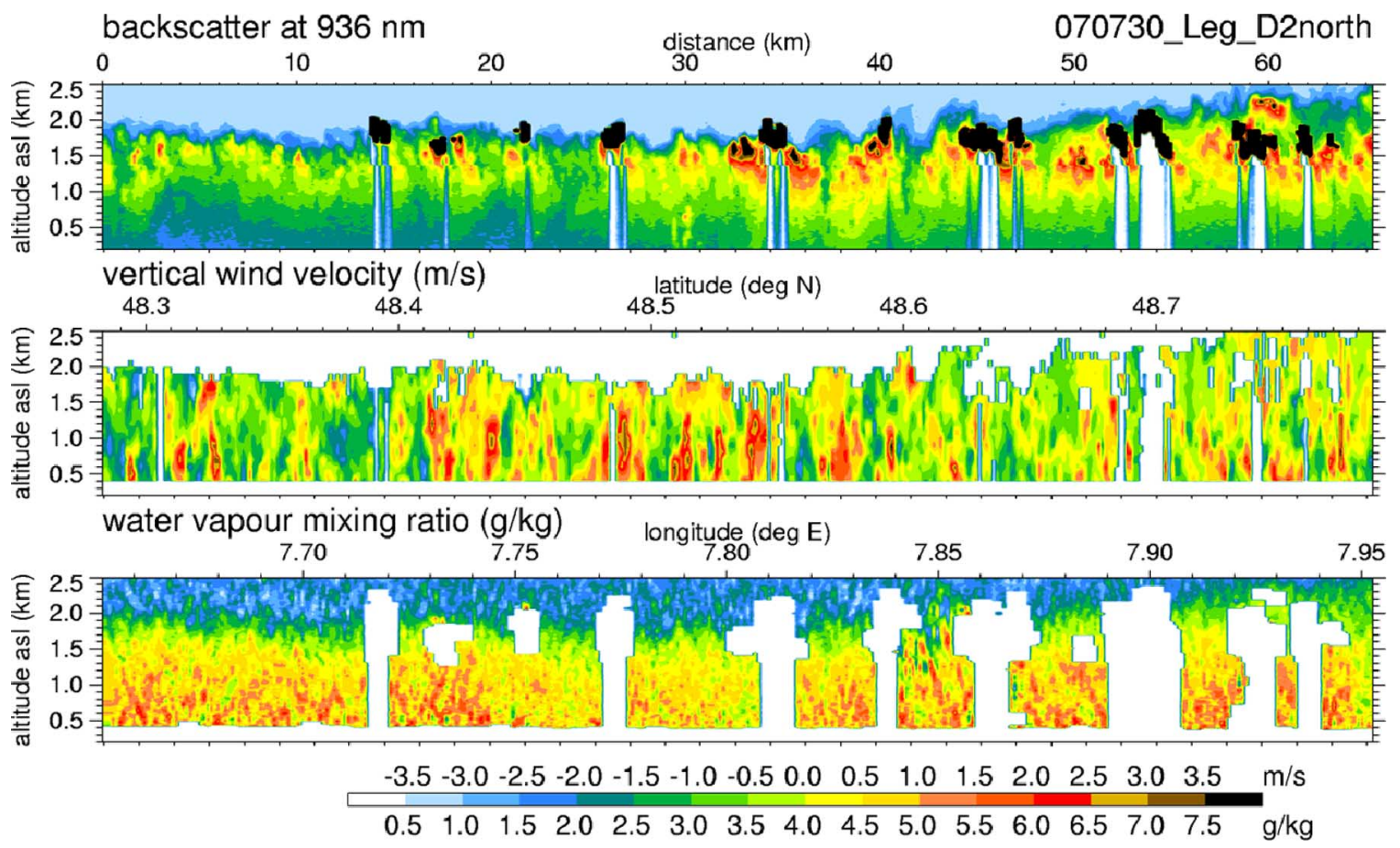

Fig. 3. Aerosol backscatter intensity (in arbitrary units; top), vertical motion (updrafts positive; middle), and humidity (bottom) above the Rhine Valley on 30 July 2007 at about local noon. The boundary layer is characterized by strong lidar backscatter intensity (green and red) due to aerosols lifted from the surface. Weak backscatter and less humidity (blue) typify the free troposphere above. Since the relative humidity increases with height in the boundary layer, the backscatter intensity increases due to swelling of aerosols, and scattered fair-weather cumulus clouds (black) form at the boundary layer top. The average boundary layer depth is $\sim 2 \mathrm{~km}$ in this case. The aspect ratio is 1:4, i.e., natural slopes and structures are squeezed horizontally. Convection, clouds, and surface influences produce an irregularly-structured boundary layer.

a)

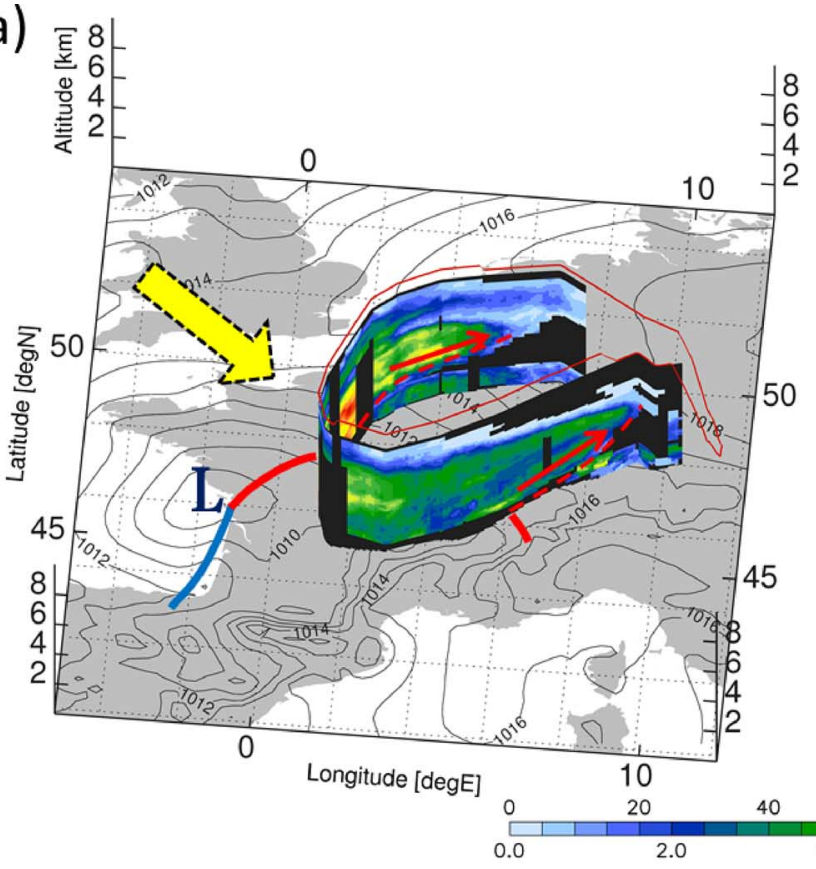

b)

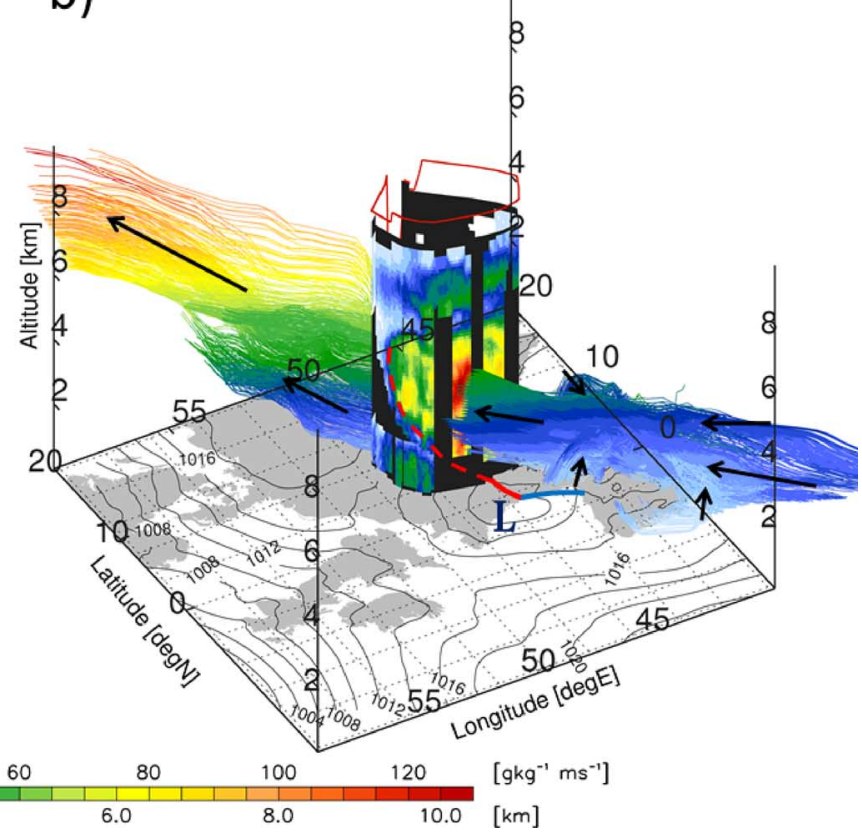

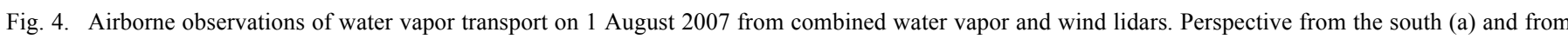
northwest (b), indicated by the yellow arrow in (a). The aircraft flew along the thin red line. The curtain-like 2-dimensional cross-sections exemplify the transport of water vapor beneath the aircraft flight track. Blue represents weak transport; yellow and red mean strong transport. Thin black contour lines on the map show the surface pressure $[\mathrm{hPa}$. The thick blue and red lines depict the location of the surface cold and warm front, respectively. The dashed red lines show the vertical extension of the warm front along the lidar curtain. In addition, (b) shows selected trajectories of air parcels with the maximum observed water vapor transport. Their colors indicate the altitude of the parcels for 2.5 days in advance and 1.5 days after the flight. Data gaps are black. 


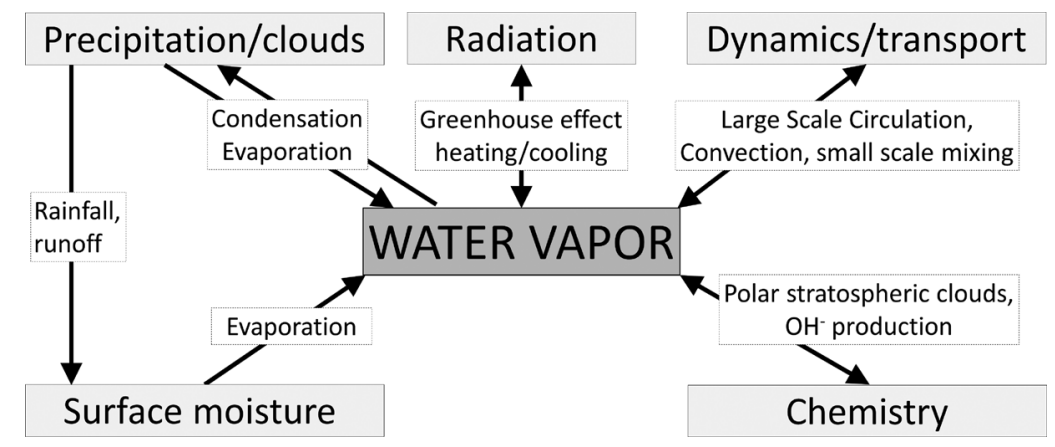

Fig. 5. Interactions of water vapor with components of the climate system. The hydrological cycle is sketched on the left. The figure is taken from [6] where more details can be found.

masses between troposphere and stratosphere. The tropopause region is very sensitive in the context of climate change due to complex feedback mechanisms between these radiation-absorbing trace gases, ice clouds, and atmospheric circulations. Last but not least, planned satellite missions to measure wind (ESA's Atmospheric Dynamics Mission), aerosols (ESA's EarthCare mission) or water vapor (ESA WALES: Water vapor Lidar Experiment in Space) can optimally be prepared and validated with airborne lidars.

\section{REFERENCES}

[1] M. Wirth, A. Fix, P. Mahnke, H. Schwarzer, F. Schrandt, and G. Ehret, "The airborne multi-wavelength water vapor differential absorption lidar WALES: System design and performance," Appl. Phys. B, vol. 96, pp. 201-213, 2009, DOI 10.1007/s00340-009-3365-7.

[2] C. Kiemle, M. Wirth, A. Fix, S. Rahm, U. Corsmeier, and P. Di Girolamo, "Latent heat flux measurements over complex terrain by airborne water vapour and wind lidars," Q. J. R. Meteorol. Soc., vol. 137, pp. 190-203, 2011.

[3] A. Schäfler, A. Dörnbrack, C. Kiemle, S. Rahm, and M. Wirth, "Tropospheric water vapor transport as determined from airborne lidar measurements," J. Atmos. Ocean. Technol., vol. 27, pp. 2017-2030, 2010.

[4] C. Kiemle, M. Wirth, A. Fix, G. Ehret, U. Schumann, T. Gardiner, C. Schiller, N. Sitnikov, and G. Stiller, "First airborne water vapor lidar measurements in the tropical upper troposphere and mid-latitudes lower stratosphere: Accuracy evaluation and intercomparisons with other instruments," Atmos. Chem. Phys., vol. 8, pp. 5245-5261, 2008.

[5] G. Poberaj, A. Fix, A. Assion, M. Wirth, C. Kiemle, and G. Ehret, "Airborne all-solid-state DIAL for water vapour measurements in the tropopause region: System description and assessment of accuracy. Special Issue "Trends in laser sources, spectroscopic techniques and their applications to trace gas detection"," Appl. Phys. B, vol. 75, pp. 165-172, 2002.
[6] C. Kiemle, A. Schäfler, and C. Voigt, "Detection and analysis of water vapor transport," in Atmospheric Physics, Research Topics in Aerospace, U. Schumann, Ed. Berlin Heidelberg: Springer-Verlag, 2012.

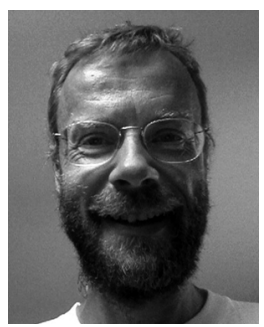

Christoph Kiemle studied electrical engineering at the Technical University of Munich and received his diploma in 1987. He started as engineer in the Lidar division of the DLR Institute of Atmospheric Physics in 1989, and became responsible for the scientific analysis of lidar data obtained during research flights with DLR aircraft. A Ph.D. in meteorology from the University of Innsbruck, Austria, followed in 2008 on the theme "Airborne Water Vapor Lidar Measurements". Current work also comprises feasibility studies for spaceborne lidar monitoring of greenhouse gases, and a lecture at the Technical University of Munich.

Andreas Schäfler, photograph and biography not available at the time of publication.

Martin Wirth, photograph and biography not available at the time of publication.

Andreas Fix, photograph and biography not available at the time of publication.

Stephan Rahm, photograph and biography not available at the time of publication. 